\title{
Fano manifolds obtained by blowing up along curves with maximal Picard number
}

\author{
Toru Tsukioka
}

November 26, 2018

\begin{abstract}
The Picard number of a Fano manifold $X$ obtained by blowing up a curve in a smooth projective variety is known to be at most 5 , in any dimension greater than or equal to 4 . We show that the Picard number attains to the maximal if and only if $X$ is the blow-up of the projective space whose center consists of two points, the strict transform of the line joining them and a linear space or a quadric of codimension 2 . This result is obtained as a consequence of a classification of special types of Fano manifolds.
\end{abstract}

\section{Introduction}

Let $X$ be a Fano manifold obtained by blowing up along a curve, i.e. there exists a pair $(Y, C)$ of a smooth projective variety $Y$ and a smooth connected curve $C \subset Y$ such that the anticanonical divisor $-K_{X}$ is ample. Using a recent result on Minimal Model Program due to [2], C. Casagrande shows that such a Fano manifold has Picard number at most 5 (see [5] Theorem 4.2 for a more general statement, and see [7] for the toric case).

The purpose of the present paper is to classify the maximal case:

Theorem 1. Let $Y$ be a smooth projective variety of dimension $n \geq 4$ defined over the field of complex numbers, $C$ a smooth curve on $Y$, and $X$ the blow-up of $Y$ along $C$. Assume that $X$ is a Fano manifold and has Picard number 5. Then, the pair $(Y, C)$ is exactly one of the following:

1. $Y$ is the blow-up of $\mathbb{P}^{n}$ whose center is the union of two points $p, q$ and $\mathbb{P}^{n-2}$ disjoint from $\overline{p q}$, and $C$ is the strict transform of $\overline{p q}$,

2. $Y$ is the blow-up of $\mathbb{P}^{n}$ whose center is the union of two points $p, q$ and a smooth quadric $Q_{n-2}$ disjoint from $\overline{p q}$, and $C$ is the strict transform of $\overline{p q}$.

Remark. We denote by $\overline{p q}$ the line passing through $p$ and $q$ in $\mathbb{P}^{n}$.

According to Casagrande's result (see [5] Theorem 4.2 (ii)), if the assumption of Theorem 1 is satisfied, then there exists another structure of blow-up $\varphi: X \rightarrow Z$ with the following properties: 
- $Z$ is a smooth projective variety, and the center of the blow-up $\varphi$ is a smooth subvariety of codimension 2

- $E \cdot f>0$, where $E$ is the exceptional divisor of the blow-up $\pi: X \rightarrow Y$ and $f$ is a non trivial fiber of $\varphi$

- $F \cdot e=0$, where $F$ is the exceptional divisor of $\varphi$ and $e$ is a line in a fiber of the $\mathbb{P}^{n-2}$-bundle $\left.\pi\right|_{E}: E \rightarrow C$

Hence, our Theorem 1 is a consequence of the following classification result (in which only two examples (8) and (9) have Picard number 5):

Theorem 2. Let $Y$ be a complex manifold of dimension $n \geq 4$. Assume that there exists a smooth curve $C \subset Y$ such that the blow-up $X$ of $Y$ along $C$ is a Fano manifold. Assume moreover that there exists a smooth projective variety $Z$ and a smooth subvariety $W \subset Z$ of codimension 2 such that the blow-up of $Z$ along $W$ is isomorphic to $X$. Let $E$ (resp. $F$ ) be the exceptional divisor of the blow-up $\pi: X \rightarrow Y$ (resp. $\varphi: X \rightarrow Z$ ). Let e (resp. $f$ ) be a line in a fiber of the $\mathbb{P}^{n-2}$-bundle $\left.\pi\right|_{E}: E \rightarrow C$ (resp. a fiber of the $\mathbb{P}^{1}$-bundle $\left.\left.\varphi\right|_{F}: F \rightarrow W\right)$. If $E \cdot f>0$ and $F \cdot e=0$, then we have exactly one of the following:

1. $Y$ is the blow-up of $\mathbb{P}^{n}$ at a point $p$ and $C$ is the strict transform of the line passing through $p$,

2. $Y$ is the blow-up of $Q_{n}$ at a point $p$ and $C$ is the strict transform of a line passing through $p$,

3. $Y$ is the blow-up of $Q_{n}$ at a point $p$ and $C$ is the strict transform of a conic passing through $p$,

4. $Y$ is the blow-up of $\mathbb{P}^{n}$ whose center is the union of a point $p$ and a linear subspace $P \simeq \mathbb{P}^{n-2}$ not containing $p$, and $C$ is the strict transform of a line passing through $p$ and disjoint from $P$,

5. $Y$ is the blow-up of $\mathbb{P}^{n}$ whose center is the union of a smooth quadric $Q \simeq Q_{n-2}$ and a point $p$ not on the hyperplane containing $Q$, and $C$ is the strict transform of a line passing through $p$ and disjoint from $Q$,

6. $Y$ is the blow-up of $\mathbb{P}^{1} \times \mathbb{P}^{n-1}$ at a point $p$ and $C$ is the strict transform of the fiber of the projection $\mathbb{P}^{1} \times \mathbb{P}^{n-1} \rightarrow \mathbb{P}^{n-1}$ passing through $p$,

7. $Y$ is the blow-up of $\mathbb{P}^{n}$ whose center is two distinct points $p$ and $q$, and $C$ is the strict transform of the line $\overline{p q}$,

8. $Y$ is the blow-up of $\mathbb{P}^{n}$ whose center is the union of two points $p, q$ and $\mathbb{P}^{n-2}$ disjoint from $\overline{p q}$, and $C$ is the strict transform of $\overline{p q}$,

9. $Y$ is the blow-up of $\mathbb{P}^{n}$ whose center is the union of two points $p, q$ and a smooth quadric $Q_{n-2}$ disjoint from $\overline{p q}$, and $C$ is the strict transform of $\overline{p q}$.

Remark. We do not assume the projectivity of $Y$ because it follows from the assumption (see Lemma 3 below). 


\section{Preliminaries}

We prove lemmas which will be needed for the proof of Theorem 2 ,

Lemma 1. We have $E \cdot f=1$.

Proof. Since $F \cdot e=0$, (the reduced part of) the intersection $E \cap F$ is a union of fibers of $\left.\pi\right|_{E}: E \rightarrow C$. Hence $E \cap F$ is the exceptional locus of $\left.\pi\right|_{F}: F \rightarrow \pi(F)$. Since $\left.\varphi\right|_{F}: F \rightarrow W$ is a $\mathbb{P}^{1}$-bundle, we see that $E \cap F$ is a section of $\left.\varphi\right|_{F}$. Hence we can write $\left.E\right|_{F}=m E_{c}$ where $m$ is a natural number and $E_{c}:=\pi^{-1}(c)$ with $c \in C$ is a fiber of $\left.\pi\right|_{E}$. Let $e_{c}$ be a line in $E_{c} \simeq \mathbb{P}^{n-2}$. We have

$$
m E_{c} \cdot e_{c}=\left.E\right|_{F} \cdot e_{c}=E \cdot e_{c}=-1
$$

where the first and second intersection numbers are taken in $F$ and the last one is in $X$. Note that $\left(E_{c} \cdot e_{c}\right)$ is an integer because $F$ is smooth. Thus we get $m=1$. It follows that $E \cap F$ is a reduced section of $\left.\varphi\right|_{F}: F \rightarrow W$. Therefore, we have $E \cdot f=1$.

Now we consider $F_{Y}:=\pi(F) \subset Y$. Note that we have $\pi^{*} F_{Y}=F$.

Lemma 2. We have $F_{Y} \cdot C=1$.

Proof. Let $\widetilde{C}$ be a section of $\left.\pi\right|_{E}: E \rightarrow C$. By (the proof of) Lemma 1, $\left.F\right|_{E}$ is a reduced fiber of $\left.\pi\right|_{E}$. Thus we have $F_{Y} \cdot C=F_{Y} \cdot \pi_{*} \widetilde{C}=F \cdot \widetilde{C}=1$.

By the proof of Lemma 1, we see that the intersection number $E_{c} \cdot e_{c}$ (taken in $F$ ) is equal to -1 . It follows that $\left.\pi\right|_{F}: F \rightarrow F_{Y}$ is the blow-up at the point $c$ whose exceptional divisor is $E_{c}$ and $F_{Y}$ is smooth. By the lemma 1, we see that $W$ is isomorphic to $E_{c} \simeq \mathbb{P}^{n-2}$. We have the diagram:

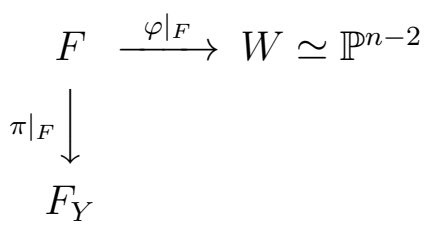

where $\left.\varphi\right|_{F}$ is a $\mathbb{P}^{1}$-bundle. Note that $F$ is a Fano manifold. Indeed, we have $\rho(F)=2$ and $F$ has two extremal contractions $\left.\pi\right|_{F}$ and $\left.\varphi\right|_{F}$. According to the classification result from [3], this implies that $F_{Y}$ is isomorphic to $\mathbb{P}^{n-1}$. Furthermore, $f_{Y}:=\pi_{*} f$ is a line passing through the point $c=F_{Y} \cap C$. Note that $F_{Y} \cdot f_{Y}=F \cdot f=-1$. Hence there exists a blow-down $\varphi^{\prime}: Y \rightarrow Y^{\prime}$ contracting $F_{Y} \simeq \mathbb{P}^{n-1}$ to a smooth point $p, Y^{\prime}$ being (a priori) a complex manifold. Hence we have the commutative diagram:

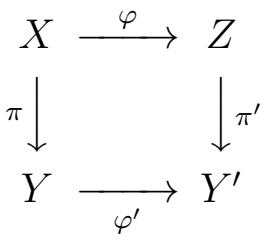

where $\pi^{\prime}: Z \rightarrow Y^{\prime}$ is the blow-up along the curve $C^{\prime}:=\varphi^{\prime}(C)$.

Lemma 3. $Y$ is projective (hence, so is $Y^{\prime}$ ). 
Proof. Assume to the contrary that $Y$ is not projective. Then, by [4] the normal bundle $N_{C / Y}$ is isomorphic to $\mathcal{O}_{\mathbb{P}^{1}}(-1)^{\oplus(n-1)}$. Since $\varphi^{\prime}: Y \rightarrow Y^{\prime}$ is a blow-up, $Y^{\prime}$ is not projective either. Note that $Z$ is projective by the assumption of Theorem 2. Hence $N_{C^{\prime} / Y^{\prime}} \simeq$ $\mathcal{O}_{\mathbb{P}^{1}}(-1)^{\oplus(n-1)}$ by [4] again. On the other hand, we have $N_{C / Y} \neq N_{C^{\prime} / Y^{\prime}}$ because $F_{Y} \cdot C>$ 0 . Hence we get a contradiction.

We recall here the classification result due to [3] which is indispensable to the proof of our Theorem 2, Let $V_{d}$ denote the blow-up of $\mathbb{P}^{n}$ along a smooth complete intersection $U_{d}:=H \cap D$ where $H$ is a hyperplane and $D$ is a hypersurface of degree $d$.

Theorem 3 (Bonavero, Campana and Wiśniewski 3] Theorem 1.1). Let $Y^{\prime}$ be a complex manifold of dimension $n \geq 3$. Let $\varphi^{\prime}: Y \rightarrow Y^{\prime}$ be the blow-up at a point $p \in Y^{\prime}$. Then $Y$ is a Fano manifold if and only if $Y^{\prime}$ is isomorphic to either $\mathbb{P}^{n}, Q_{n}$, or $V_{d}$ with $1 \leq d \leq n$ and $p$ is not on the hyperplane $H$ containing the center $U_{d}$.

\section{Proof of Theorem 2}

The proof is divided into two parts:

(A) If $Y$ is a Fano manifold, then $(Y, C)$ is one of the examples from (1) to (5).

(B) If $Y$ is not a Fano manifold, then $(Y, C)$ is one of the examples from (6) to (9).

Throughout the section, we frequently use the following:

Lemma 4 (cf.[6] Proposition 7). Let $Y$ be a smooth projective variety of dimension $n \geq 3$, $C \subset Y$ a smooth subvariety of codimension $k \geq 2$, and $X$ the blow-up of $Y$ along $C$. Assume that $X$ is a Fano manifold. If $\Gamma \subset Y$ is a curve not contained in $C$ and $\Gamma \cap C \neq \varnothing$, then we have $\left(-K_{Y}\right) \cdot \Gamma \geq k$.

Proof. Let $\widetilde{\Gamma}$ be the strict transform of $\Gamma$ by the blow-up. For the exceptional divisor $E$, we have $E \cdot \widetilde{\Gamma} \geq 1$. Hence we have

$$
0<-K_{X} \cdot \widetilde{\Gamma}=-K_{Y} \cdot \Gamma-(k-1) E \cdot \widetilde{\Gamma} \leq-K_{Y} \cdot \Gamma-(k-1),
$$

which gives the statement.

In what follows, we use the notation of the diagram (1) in the previous section.

\subsection{Proof of (A)}

We assume that $Y$ is a Fano manifold. Since $\varphi^{\prime}: Y \rightarrow Y^{\prime}$ is a blow-up at a point, we are exactly in the situation of Theorem 3. Consider the extremal contraction $\gamma: Y \rightarrow Y^{\prime \prime}$ of ray $\mathbb{R}^{+}[g]$ such that $F_{Y} \cdot g>0$ (see [3] Lemme 2.1 for the existence of such a contraction). Then, by [3] Proposition 2.2, $\gamma$ is either:

(A1) a $\mathbb{P}^{1}$-bundle, or

(A2) a blow-up of a smooth projective variety along a smooth subvariety of codimension 2 . 
In the case (A1), $Y^{\prime}$ is isomorphic to $\mathbb{P}^{n}$. We shall determine the position of $C$ in $Y$. If $C$ is not a fiber of $\gamma$, then there exists a fiber $\Gamma \simeq \mathbb{P}^{1}$ of $\gamma$ such that $\Gamma \cap C \neq \varnothing$. Note that $-K_{Y} \cdot \Gamma=2$. Hence, by Lemma 4 this is a contradiction. It follows that $C$ is a fiber of $\gamma$, i.e. the strict transform of a line in $Y^{\prime} \simeq \mathbb{P}^{n}$ passing through $p$, the center of the blow-up $\varphi^{\prime}$. So, we get the example (1).

Now we treat the case (A2). Let $W_{\gamma}$ be the center of the blow-up $\gamma: Y \rightarrow Y^{\prime \prime}$ and $G$ the exceptional divisor. Note that $\left.\gamma\right|_{G}: G \rightarrow W_{\gamma}$ is a $\mathbb{P}^{1}$-bundle. By [3], there are two possibilities:

(A2-1) $Y^{\prime \prime}$ is isomorphic to $\mathbb{P}^{n}$ and $W_{\gamma}$ is isomorphic to $Q_{n-2}$, or

(A2-2) $Y^{\prime \prime}$ is isomorphic to the $\mathbb{P}^{1}$-bundle $\mathbb{P}\left(\mathcal{O}_{\mathbb{P}^{n-1}} \oplus \mathcal{O}_{\mathbb{P}^{n-1}}(d-1)\right)$ and $W_{\gamma}$ is a hypersurface in the section $\mathbb{P}\left(\mathcal{O}_{\mathbb{P}^{n-1}}\right) \simeq \mathbb{P}^{n-1}$ whose normal bundle is isomorphic to $\mathcal{O}_{\mathbb{P}^{n-1}}(d-1)$.

In the case $(\mathrm{A} 2-1), Y^{\prime}$ is isomorphic to $Q_{n}$. Note that $F_{Y}=\operatorname{Exc}\left(\varphi^{\prime}\right)$ is the strict transform of the hyperplane containing $W \simeq Q_{n-2}$ by the blow-up $\gamma: Y \rightarrow Y^{\prime \prime} \simeq \mathbb{P}^{n}$. Since $F_{Y} \cdot C=1$ (Lemma 2), $C$ is either a fiber of the $\mathbb{P}^{1}$-bundle $\left.\gamma\right|_{G}: G \rightarrow W$, or the strict transform of a conic passing through $p \in Q_{n}$. So, we get the examples (2) or (3).

In the case (A2-2), $Y^{\prime}$ is isomorphic to $V_{d}$ with $1 \leq d \leq n$. Let $\beta: Y^{\prime} \simeq V_{d} \rightarrow \mathbb{P}^{n}$ denote the blow-up along the smooth complete intersection $U_{d}=H \cap D$ with $H \in\left|\mathcal{O}_{\mathbb{P}^{n}}(1)\right|$ and $D \in\left|\mathcal{O}_{\mathbb{P}^{n}}(d)\right|$. Consider the composite of the two blow-ups $\varepsilon:=\varphi^{\prime} \circ \beta: Y \rightarrow \mathbb{P}^{n}$. Note that the exceptional divisor $G$ of $\gamma$ is the strict transform by $\varepsilon$ of the cone over $U_{d}$ with vertex $\beta(p)$ (recall that $p$ is the center of the blow-up $\varphi^{\prime}: Y \rightarrow Y^{\prime}$ ). Let $H_{Y}$ be the strict transform by $\varepsilon$ of the hyperplane $H$ containing $U_{d}$. We have $H_{Y} \cap F_{Y}=\varnothing$ because $\varepsilon\left(F_{Y}\right) \notin H$ (see the statement of Theorem 3).

Claim 1. We have $H_{Y} \cdot C=1$.

Proof. Let $M$ be the exceptional divisor of the blow-up $\beta$ and $M_{Y}$ its strict transform by $\varphi^{\prime}$. Since $F_{Y} \cdot C=1$ and $M_{Y} \cap F_{Y}=\varnothing$, we see that $C \not \subset M_{Y}$. In particular we have $\varepsilon_{*} C \not \equiv 0$. If $C \cap M_{Y} \neq \varnothing$, then there exists a fiber $\Gamma$ of the $\mathbb{P}^{1}$-bundle $M_{Y} \rightarrow U_{d}$ meeting $C$. Note that $-K_{Y} \cdot \Gamma=1$. By Lemma 4, this is a contradiction. Hence $M_{Y} \cdot C=0$. Note that $\varepsilon^{*} H=H_{Y}+M_{Y}$. We have

$$
H_{Y} \cdot C=\left(H_{Y}+M_{Y}\right) \cdot C=\varepsilon^{*} H \cdot C=H \cdot \varepsilon_{*} C>0 .
$$

If $H_{Y} \cdot C \geq 2$ then there exists a line $h \subset H_{Y} \simeq \mathbb{P}^{n-1}$ whose strict transform $\widetilde{h}$ by the blow-up $\pi: X \rightarrow Y$ satisfies $E \cdot \widetilde{h} \geq 2$. Then we have

$$
K_{X} \cdot \widetilde{h}=K_{Y} \cdot h+(n-2) E \cdot \widetilde{h} \geq-n+d-1+2(n-2)=n+d-5 \geq 0,
$$

which is a contradiction because $X$ is a Fano manifold. Hence we are done.

Claim 2. We have $d=1$ or 2 .

Proof. Let $h$ be a line in $H_{Y} \simeq \mathbb{P}^{n-1}$ such that $E \cdot \widetilde{h}=1$. Then we have

$$
K_{X} \cdot \widetilde{h}=K_{Y} \cdot h+(n-2) E \cdot \widetilde{h}=d-3 .
$$

Since $K_{X} \cdot \widetilde{h}<0$, we get $d=1$ or 2 .

If $d=1$, we get the example (4) and if $d=2$, the example (5). The curve $C$ is determined by the condition $H_{Y} \cdot C=1$ and $F_{Y} \cdot C=1$. 


\subsection{Proof of (B)}

Assume that $Y$ is not a Fano manifold. By [9] Proposition 3.5, $E$ is isomorphic to $\mathbb{P}^{1} \times \mathbb{P}^{n-2}$ and $E \cdot l=-1$ where $l$ is a fiber of the projection $E \simeq \mathbb{P}^{1} \times \mathbb{P}^{n-2} \rightarrow \mathbb{P}^{n-2}$. Put $E_{Z}:=\varphi(E), e_{Z}:=\varphi_{*} e$ and $l_{Z}:=\varphi_{*} l$. Since $E \cdot f=1,\left.\varphi\right|_{E}: E \rightarrow E_{Z}$ is an isomorphism. Since $\varphi^{*} E_{Z}=E+F$, we have $E_{Z} \cdot e_{Z}=-1$ and $E_{Z} \cdot l_{Z}=0$. Recall that $Y^{\prime}$ is projective by Lemma 3 .

Lemma 5. The projective varieties $Y^{\prime}$ and $Z$ are Fano manifolds.

Proof. Since $E_{Z} \cdot l_{Z}=0$, we have $N_{C^{\prime} / Y^{\prime}} \simeq \mathcal{O}_{\mathbb{P}^{1}}^{\oplus(n-1)} \not \mathcal{O}_{\mathbb{P}^{1}}(-1)^{\oplus(n-1)}$. Therefore, $Y^{\prime}$ is a Fano manifold by [9] Proposition 3.5. Since $K_{X}=\varphi^{*} K_{Z}+F$, we get

$$
\left(-K_{Z}\right) \cdot e_{Z}=-K_{X} \cdot e+F \cdot e=-K_{X} \cdot e>0 .
$$

Note that the center $W$ of the blow-up $\varphi: X \rightarrow Z$ is a fiber of the projection $E_{Z} \simeq$ $\mathbb{P}^{1} \times \mathbb{P}^{n-2} \rightarrow \mathbb{P}^{1}$. Hence, any curve contained in $W$ is numerically proportional to a positive multiple of the line $e_{Z}$. By [8] Proposition 1, we conclude that $Z$ is a Fano manifold.

Since $Z$ is a Fano manifold, there exists an extremal ray $\mathbb{R}^{+}[m] \subset \overline{\mathrm{NE}}(Z)$ such that $E_{Z} \cdot m>0$ (see [3] Lemme 2.1). We investigate the associated extremal contraction $\mu: Z \rightarrow Z^{\prime}$.

Lemma 6. We have $\mu_{*} B \not \equiv 0$ for any curve $B$ contained in $E_{Z}$.

Proof. Assume to the contrary that there exists a curve $B \subset E_{Z}$ such that $\mu_{*} B \equiv 0$. Then, there exists $a>0$ such that $B \equiv a m$. On the other hand, we can write $B \equiv b l_{Z}+c e_{Z}$ with $b, c \geq 0$ because $B$ is contained in $E_{Z} \simeq \mathbb{P}^{1} \times \mathbb{P}^{n-2}$. So, we have $E_{Z} \cdot B=E_{Z} \cdot($ am $)>0$ and $E_{Z} \cdot B=E_{Z} \cdot\left(b l_{Z}+c e_{Z}\right)=-c \leq 0$, a contradiction.

If there exists $z^{\prime} \in Z^{\prime}$ such that $\operatorname{dim} \mu^{-1}\left(z^{\prime}\right) \geq 2$, then there exists a curve $B$ contained in $E_{Z} \cap \mu^{-1}\left(z^{\prime}\right)$. Hence, any non-trivial fiber of $\mu$ has dimension at most 1. By [1] (see [9] Theorem 1.2), the extremal contraction $\mu$ is either:

(B1) a conic bundle, or

(B2) a blow-up of a smooth projective variety along a smooth subvariety of codimension 2 .

First we treat the case (B1). We show that $\mu$ has no singular fiber, i.e. $\mu$ is a $\mathbb{P}^{1}$ bundle. Let $\Gamma$ be a fiber of $\mu$. Note that $\Gamma$ is isomorphic to $\mathbb{P}^{1}$ or $\Gamma \simeq \Gamma_{1} \cup \Gamma_{2}$ with $\Gamma_{i} \simeq \mathbb{P}^{1}(i=1,2)$.

Claim 3. If $\Gamma$ meets $W$, then $\Gamma$ is a smooth fiber and the intersection $\Gamma \cap W$ is one point with multiplicity one.

Proof. Assume $\Gamma=\Gamma_{1} \cup \Gamma_{2}$ with $\Gamma_{1} \cap W \neq \varnothing$. Note that $-K_{Z} \cdot \Gamma_{1}=1$. By Lemma 4, this is a contradiction. Hence $\Gamma$ is a smooth fiber. Let $\widetilde{\Gamma}$ be the strict transform by $\varphi$. We have

$$
0<-K_{X} \cdot \widetilde{\Gamma}=-K_{Z} \cdot \Gamma-F \cdot \widetilde{\Gamma}=2-F \cdot \widetilde{\Gamma},
$$

which gives $F \cdot \widetilde{\Gamma}=1$ and completes the proof. 
We conclude that $\left.\mu\right|_{W}: W \rightarrow \mu(W)$ is an isomorphism. In particular, $\mu(W) \simeq \mathbb{P}^{n-2}$. We put $M:=\mu^{-1}(\mu(W))$. Remark that $\left.\mu\right|_{M}: M \rightarrow \mu(W)$ is a $\mathbb{P}^{1}$-bundle and $W$ is a section.

Claim 4. We have $E_{Z} \cdot \Gamma=1$.

Proof. By Claim 3, it is sufficient to prove $E_{Z} \cap M=W$. Let $\Gamma$ be any fiber of $\left.\mu\right|_{M}$ : $M \rightarrow \mu(W)$. We show that $E_{Z} \cap \Gamma \subset W$. Assume to the contrary that there exists a point $z \in E_{Z} \cap \Gamma$ such that $z \notin W$. Let $\Phi$ be the fiber of the $\mathbb{P}^{n-2}$-bundle $\left.\pi^{\prime}\right|_{E_{Z}}: E_{Z} \rightarrow C^{\prime}$ containing the point $z$. Since $\operatorname{dim} M \cap \Phi=n-3 \geq 1$, there exists a curve $A \subset M \cap \Phi$. Consider the ruled surface $S:=\mu^{-1}(\mu(A))$. By Lemma 6 above, $\Gamma \not \subset E_{Z}$, hence $\pi^{\prime}(\Gamma) \not \subset C^{\prime}$ and we have $\operatorname{dim} \pi^{\prime}(S)=2$. Therefore, $W \cap S$ and $A$ are exceptional curves on $S$. Note that $A \neq W \cap S$ because $\Phi \cap W=\varnothing$. Thus, we have a contradiction because $S$ is a ruled surface.

Now, we see that $\mu: Z \rightarrow Z^{\prime}$ is a $\mathbb{P}^{1}$-bundle and $\left.\mu\right|_{E_{Z}}: E_{Z} \rightarrow Z^{\prime}$ is an isomorphism. It follows that $Z^{\prime}$ is isomorphic to $\mathbb{P}^{1} \times \mathbb{P}^{n-2}$. Pushing down the exact sequence:

$$
0 \rightarrow \mathcal{O}_{Z} \rightarrow \mathcal{O}_{Z}\left(E_{Z}\right) \rightarrow \mathcal{O}_{E_{Z}}\left(E_{Z}\right) \rightarrow 0,
$$

we get

$$
0 \rightarrow \mu_{*} \mathcal{O}_{Z} \rightarrow \mu_{*} \mathcal{O}_{Z}\left(E_{Z}\right) \rightarrow \mu_{*} \mathcal{O}_{E_{Z}}\left(E_{Z}\right) \rightarrow R^{1} \mu_{*} \mathcal{O}_{Z}=0 .
$$

Since $\mu$ is an extremal contraction, we have $\mu_{*} \mathcal{O}_{Z} \simeq \mathcal{O}_{\mathbb{P}^{1} \times \mathbb{P}^{n-2}}$. Recall that $\mathcal{O}_{E_{Z}}\left(E_{Z}\right) \simeq$ $\mathcal{O}_{\mathbb{P}^{1} \times \mathbb{P}^{n-2}}(0,-1)$. Since $\left.\mu\right|_{E_{Z}}$ is an isomorphism, we have $\mu_{*} \mathcal{O}_{E_{Z}}\left(E_{Z}\right) \simeq \mathcal{O}_{\mathbb{P}^{1} \times \mathbb{P}^{n-2}}(0,-1)$. Thus we get the splitting sequence

$$
0 \rightarrow \mathcal{O}_{\mathbb{P}^{1} \times \mathbb{P}^{n-2}} \rightarrow \mu_{*} \mathcal{O}_{Z}\left(E_{Z}\right) \rightarrow \mathcal{O}_{\mathbb{P}^{1} \times \mathbb{P}^{n-2}}(0,-1) \rightarrow 0,
$$

which gives $\mu_{*} \mathcal{O}_{Z}\left(E_{Z}\right) \simeq \mathcal{O}_{\mathbb{P}^{1} \times \mathbb{P}^{n-2}} \oplus \mathcal{O}_{\mathbb{P}^{1} \times \mathbb{P}^{n-2}}(0,-1)$. Thus we have

$$
Z \simeq \mathbb{P}\left(\mathcal{O}_{\mathbb{P}^{1} \times \mathbb{P}^{n-2}} \oplus \mathcal{O}_{\mathbb{P}^{1} \times \mathbb{P}^{n-2}}(0,-1)\right) \simeq \mathbb{P}^{1} \times \mathrm{Bl}_{p}\left(\mathbb{P}^{n-1}\right),
$$

where $\mathrm{Bl}_{p}\left(\mathbb{P}^{n-1}\right)$ denotes the blow-up of $\mathbb{P}^{n-1}$ at the point $p$. We see that $Y^{\prime} \simeq \mathbb{P}^{1} \times \mathbb{P}^{n-1}$ and $C^{\prime}$ is a fiber of the projection $Y^{\prime} \rightarrow \mathbb{P}^{n-1}$. We obtain the example (6).

Now we consider the case (B2). Let $F_{Z}$ be the exceptional divisor of the blow-up $\mu: Z \rightarrow Z^{\prime}$. Since $E_{Z}$ is strictly positive on the extremal ray $\mathbb{R}^{+}[m]$, we have $E_{Z} \neq F_{Z}$, in particular $F_{Z} \cdot e_{Z} \geq 0$. If $F_{Z} \cdot e_{Z}>0$, there exists a fiber $m_{0}$ of the $\mathbb{P}^{1}$-bundle $F_{Z} \rightarrow \mu\left(F_{Z}\right)$ such that $m_{0} \cap W \neq \varnothing$ (recall that $W$ denote the center of the blow-up $\varphi: X \rightarrow Z$ ). Since $-K_{Z} \cdot m_{0}=1$, we get a contradiction by Lemma 4. Hence we have $F_{Z} \cdot e_{Z}=0$.

Recall that $\pi^{\prime}: Z \rightarrow Y^{\prime}$ is the blow-up along $C^{\prime}$ and $\mu: Z \rightarrow Z^{\prime}$ is a blow-up along a center of codimension 2 with $F_{Z} \cdot e_{Z}=0$. Since $Y^{\prime}$ and $Z$ are Fano manifolds, we can use the statement (A) (already proved in the previous subsection) to classify the pairs $\left(Y^{\prime}, C^{\prime}\right)$. Moreover, we have the condition on the normal bundle: $N_{C^{\prime} / Z^{\prime}} \simeq \mathcal{O}_{\mathbb{P}^{1}}^{\oplus(n-1)}$, which is satisfied for the following cases:

- $Y^{\prime}$ is the blow-up of $\mathbb{P}^{n}$ at a point $q$ and $C^{\prime}$ is the strict transform of the line passing through $q$ 
- $Y^{\prime}$ is the blow-up of $\mathbb{P}^{n}$ at a point $q$ and a linear subspace $P \simeq \mathbb{P}^{n-2}$ and $C^{\prime}$ is the strict transform of a line passing through $q$,

- $Y^{\prime}$ is the blow-up of $\mathbb{P}^{n}$ at a point $q$ and a quadric $Q \simeq Q_{n-2}$ and $C^{\prime}$ is the strict transform of a line passing through $q$.

Recall that in each case, $Y$ is the blow-up of $Y^{\prime}$ at the point $p \in C^{\prime}$. So, we get the examples (7), (8), and (9). Hence, the proof of the statement (B) is completed.

\section{References}

[1] T. Ando, On extremal rays of the higher-dimensional varieties. Invent. Math. 81, (1985) 347-357.

[2] C. Birkar, P. Cascini, C. Hacon, and J. McKernan, Existence of minimal models for varieties of log general type. arXiv:math/0610203

[3] L. Bonavero, F. Campana and J. Wisniewski, Variétés complexes dont l'éclatée en un point est de Fano. C. R. Math. Acad. Sci. Paris 334, (2002) 463-468.

[4] L. Bonavero and S. Takayama, Some Boundedness Results for Fano-Like Moishezon Manifolds. Documenta Math. 5, (2000) 141-150.

[5] C. Casagrande, On Fano manifolds with a birational contraction sending a divisor to a curve. arXiv:0807.2323

[6] S. Mori and S. Mukai, Classification of Fano 3-folds with $B_{2} \geq 2$. Manuscripta Math. 36, (1981/82) 147-162. Erratum: Manuscripta Math. 110, (2003) 407.

[7] H. Sato, Toric Fano varieties with divisorial contractions to curves. Math. Nachr. 261/262, (2003) 163-170.

[8] T. Tsukioka, Classification of Fano manifolds containing a negative divisor isomorphic to projective space. Geometriae Dedicata 123, (2006) 179-186.

[9] J. Wiśniewski, On contractions of extremal rays of Fano manifolds. J. Reine Angew. Math. 417, (1991) 141-157.

Toru TSUKIOKA e-mail: tsukiokatoru@yahoo.co.jp

Faculty of Liberal arts and Sciences, Osaka Prefecture University

1-1 Gakuen-cho Nakaku Sakai, Osaka 599-8531 Japan 